\title{
CULTIVO DE Pleurotus sp. Y Lentinula edodes BAJO CONDICIONES ARTESANALES EN COMUNIDADES CAMPESINAS DE LA REGIÓN CUSCO / PERÚ
}

\section{CULTIVATION OF Pleurotus sp. AND Lentinula edodes UNDER ARTISANAL CONDITIONS IN RURAL COMMUNITIES OF THE CUSCO REGION / PERU}

María E. Holgado-Rojas ${ }^{1,2}$, Rosana L. Aranzabal Carrasco ${ }^{2,3}$, Ruth Lazarte Lovaton ${ }^{1,2}$, Albino Quispe Peláez ${ }^{1,2}$, Karin A. Pérez Leguía ${ }^{1,2}$, Frank B. Aguilar Mainicta ${ }^{1,2}$ y Fraido Aguilar Pumahuillca ${ }^{1,2}$

\section{Resumen}

El trabajo de investigación se realizó con la finalidad de evaluar la producción y el valor proteico de hongos comestibles utilizando residuos agrícolas y forestales (chala de maíz, rastrojo de trigo, cebada, aserrín de eucalipto entre otros) generados en comunidades campesinas de la región Cusco. Se determinó el Ciclo de cultivo (CC), Eficiencia Biológica (EB) y Tasa de Producción (TP). Pleurotus ostreatus presentó mayor capacidad de adaptación a los diferentes parámetros ambientales en las tres comunidades con EB que oscilaron entre 43.8-58.2\%, CC entre 42-91 días y TP entre 0.6 y $1.4 \%$, mientras que $P$. djamor y Lentinula edodes solo presentaron cuerpos fructíferos en las comunidades de Harin y San Nicolás de Bari con EB: 27.3-34.8\%, CC : 42-90 días y TP: 0.4-0.7\% para P. djamor, y EB: 40.6-51.3\%, CC: 135-155 días y TP: 0.3-0.4\% para L. edodes. El análisis de proteínas en peso seco arrojó resultados considerables para $P$. ostreatus $(35.45 \%)$ y L. edodes (34.54\%) mientras que $P$. djamor presentó $19.68 \%$. De acuerdo al sistema Pico Tag Water, $P$. ostreatus presenta ocho de los diez aminoácidos esenciales requeridos para una alimentación saludable (His, Thr, Val, Met, Ile, Leu, Phe y Lys). Las tres especies son una importante fuente de fósforo $(366.6 \mathrm{mg} / 100 \mathrm{~g})$, magnesio $(54 \mathrm{mg} / 100 \mathrm{~g})$, calcio $(16.7 \mathrm{mg} / 100 \mathrm{~g})$ y hierro $(3.6 \mathrm{mg} / 100 \mathrm{~g})$, valores promedio que superan a los reportados en la leche de vaca, hígado de pollo, y maíz blanco. Palabras clave: cultivo, hongos comestibles, comunidades campesinas, Cusco, Perú.

\begin{abstract}
The research was carried out with the purpose of evaluating the production and protein value of edible fungi using agricultural and forest residues (cornhusk, wheat stubble, barley, eucalyptus sawdust among others) generated in rural communities of the Cusco region. The crop cycle (CC), Biological Efficiency (EB) and Production Rate (TP) were determined. Pleurotus ostreatus showed greater adaptability to the different environmental parameters in the three communities with EB that ranged between 43.8-58.2\%, CC between 42 - 91 days and TP between 0.6 and 1.4\%, while $P$. djamor and Lentinula edodes only presented fruiting bodies in the communities of Harin and San Nicolás de Bari with EB: 27.3-34.8\%, CC: $42-90$ days and TP: 0.4-0.7\% for P. djamor, and EB: 40.6-51.3\%, CC: $135-155$ days and TP: $0.3-0.4 \%$ for L. edodes. The dry weight protein analysis yielded considerable results for P. ostreatus (35.45\%) and L. edodes (34.54\%) while P. djamor presented $19.68 \%$. According to the Pico Tag Water system, P. ostreatus has eight of the ten essential amino acids required for healthy eating (His, Thr, Val, Met, Ile, Leu, Phe and Lys). The three species are an important source of phosphorus (366.6 mg/100 g), magnesium (54 mg/100 g), calcium $(16.7 \mathrm{mg} / 100 \mathrm{~g})$ and iron $(3.6 \mathrm{mg} / 100 \mathrm{~g})$, average values that exceed reported in cow's milk, chicken liver, and white corn.
\end{abstract}

Key words: cultivation, edible fungi, peasant communities, Cusco, Peru.

\section{Introducción}

El cultivo de hongos en el mundo ha tenido un significativo impacto en la producción de alimentos y ha ayudado a resolver el problema de la disposición de desechos orgánicos no comestibles (Chang, 1993). Se trata de procesos biotecnológicos que pueden desarrollarse a pequeña y gran escala como una alternativa en la producción de alimentos en el medio rural que no afecta las actividades de la vida campesina y tampoco daña su entorno ecológico. En este contexto, Latinoamérica representa una región emergente con gran potencial de desarrollo en virtud de su diversidad cultural, biológica y ecológica; temas como las propiedades funcionales, medicinales $\mathrm{y}$ 
biotecnológicas de los hongos permitirán alcanzar el desarrollo sostenible, superando los grandes retos globales que representan la crisis económica y alimentaria, el cambio climático y la disminución de la disponibilidad del agua (Martínez-Carrera et al., 2010).

En el Perú, el cultivo de hongos comestibles se inició en el año 1960 con la introducción de Agaricus bisporus "champiñón"; sin embargo, no fue hasta los años 80 que el cultivo alcanzó niveles industriales. Posteriormente, en 1990, se introduce el cultivo de Pleurotus ostreatus (setas), y el 2008 se logra, por primera vez, la oferta de Lentinula edodes "shiitake" (Chimey \& Holgado, 2010).

Para desarrollar esta tecnología se utilizan desechos agrícolas y forestales que se encuentran en abundancia en áreas rurales de regiones del mundo en desarrollo, cuyas economías aún son básicamente agrícolas, donde con mucha frecuencia sufren de deficiencia proteica, por lo que el cultivo de estos hongos puede hacer importantes contribuciones en su nutrición y bienestar económico (Mshigeni \& Chang, 2017), así como ser una alternativa importante para el reciclaje de sus subproductos (Pérez-Merlo \& Mata, 2005; Vega et $a l ., 2005)$, evitando que se convierta en una fuente de contaminación y aminorando el impacto ambiental de su disposición final.

En general, los hongos comestibles contienen $90 \%$ de agua y $10 \%$ de materia seca, de los cuales $27-48 \%$ son proteína, aproximadamente $60 \%$ corresponde a carbohidratos, en especial fibras dietéticas (Dglucanas, quitina y sustancias pécticas) y 2-8\% son lípidos (Sánchez, 2004). El alto contenido proteico, (15\% al 35\% del peso seco) puede ser comparado con el de muchas especies vegetales (Kiwicha, tarwi, maíz blanco, etc). El contenido de minerales varía entre $6 \%$ y $11 \%$ según la especie; los que aparecen en mayor cantidad son el calcio, potasio, fósforo, magnesio, zinc y cobre; en cuanto al contenido de vitaminas, son ricos en riboflavina (B2), niacina (B3) y folatos (B9) (Roncero, 2015).

En el presente trabajo se evalúa la producción y el valor proteico de Pleurotus ostreatus "k'allampa" (Herrera, 1941) y Lentinula edodes "shiitake" bajo condiciones artesanales utilizando como sustrato residuos lignocelulósicos (maíz, cebada y trigo) que presentan mayor superficie cultivada en el área de estudio (MINAM, 2015), innovando las capacidades de los agricultores en esta biotecnología, la misma que puede ser replicada en otras comunidades campesinas de la Región Cusco.

\section{Metodología}

Adecuación de ambientes

El presente estudio se realizó en tres comunidades campesinas de la Región del Cusco: Huayllay (3 665 msnm, 18 L 817550.59 m E - 8498279.91 m S), San Nicolás de Bari (3 405 msnm, 18 L 797197.69 m E $8510776.75 \mathrm{~m} \mathrm{~S}$ ) y Harin (2 $929 \mathrm{msnm}, 18 \mathrm{~L} 825321.12$

m E - 8526410.39 m S). En cada comunidad se organizaron a los pobladores en asociaciones productoras, las cuales dispusieron de dos ambientes dentro de sus viviendas. El primer ambiente fue utilizado para la incubación miceliar de las unidades de cultivo, la que se mantuvo sin iluminación y con poca ventilación, mientras que el segundo se utilizó para la fructificación de los basidiocarpos; por el contrario, este ambiente estuvo iluminado durante $10+/-1$ hora (luz natural), ventilado y con una humedad relativa entre $80-90 \%$, el mismo que se mantuvo con riego constante. En ambos casos las paredes fueron previamente acondicionadas con polietileno de doble densidad de acuerdo a la etapa del cultivo; de igual forma se construyeron andamios de madera para la disposición de las unidades de investigación, mientras que para la adecuación y pasteurización de la materia prima se utilizó un ambiente comunitario. La ventaja de este sistema de cultivo (polizona) es la optimización de las condiciones para cada etapa del cultivo (Albertó, 2008).

Tabla 1. Formulación de los sustratos en las comunidades campesinas de Huayllay, Harin y San Nicolás de Bari.

\begin{tabular}{|c|c|c|}
\hline ESPECIE & COMUNIDAD & FÓRMULA \\
\hline P. ostreatus & Huayllay & $\begin{array}{l}\text { Rastrojo de cebada } \\
(60 \%)+\text { Chala de } \\
\text { maíz }(40 \%)\end{array}$ \\
\hline P. ostreatus & $\begin{array}{l}\text { San Nicolás de } \\
\text { Bari }\end{array}$ & $\begin{array}{l}\text { Chala de maíz } \\
(100 \%)\end{array}$ \\
\hline P. ostreatus & Harin & $\begin{array}{l}\text { Chala de maíz }(80 \%) \\
+ \text { Marlo de maíz } \\
(10 \%)+\text { Cascara de } \\
\text { habas }(10 \%)\end{array}$ \\
\hline P. djamor & Huayllay & $\begin{array}{l}\text { Rastrojo de cebada } \\
(60 \%)+\text { Chala de } \\
\text { maíz }(40 \%)+\text { cal } \\
(1 \%)\end{array}$ \\
\hline P. djamor & $\begin{array}{l}\text { San Nicolás de } \\
\text { Bari }\end{array}$ & $\begin{array}{l}\text { Chala de maíz (39\%) } \\
+ \text { Marlo de maíz } \\
(39 \%)+\text { Rastrojo de } \\
\text { trigo }(20 \%)+\text { cal } \\
(1 \%)\end{array}$ \\
\hline P. djamor & Harin & $\begin{array}{l}\text { Chala de maíz (80\%) } \\
+ \text { Salvado de trigo } \\
(18 \%)+\text { Cal }(1 \%)\end{array}$ \\
\hline L. edodes & Huayllay & $\begin{array}{l}\text { Aserrín de eucalipto } \\
(80 \%)+\text { Salvado de } \\
\text { trigo }(18 \%)+\text { yeso } \\
(1 \%)+\text { Cal }(1 \%)\end{array}$ \\
\hline L. edodes & $\begin{array}{l}\text { San Nicolás de } \\
\text { Bari }\end{array}$ & $\begin{array}{l}\text { Aserrín de eucalipto } \\
(80 \%)+\text { Salvado de } \\
\text { trigo }(18 \%)+\text { Yeso } \\
(1 \%)+\text { Cal }(1 \%)\end{array}$ \\
\hline L. edodes & Harin & $\begin{array}{l}\text { Aserrín de eucalipto } \\
(80 \%)+\text { Salvado de } \\
\text { trigo }(18 \%)+\text { Yeso } \\
(1 \%)+\mathrm{Cal}(1 \%)\end{array}$ \\
\hline
\end{tabular}


Inóculo fúngico (Cepas)

Se estudiaron tres cepas producidas en el Centro de Investigación y Producción de Hongos Alimenticios y Medicinales - CIPHAM de la Universidad Nacional de San Antonio Abad del Cusco - UNSAAC, usando como soporte granos de trigo:

- CIPHAM A01 Pleurotus ostreatus (Jaq ex Fr.) P. Kumm;

- CIPHAM B02 Pleurotus djamor (Rumph ex Fr.); y

- CIPHAM D01 Lentinula edodes Pegler.

$\underline{\text { Sustratos }}$

Para el cultivo de $P$. ostreatus y $P$. djamor se usaron como sustrato residuos de la actividad agrícola, mientras que para $L$. edodes se utilizó aserrín de eucalipto, tomando en cuenta las formulaciones propuestas por Martínez-Carrera et al. (1984) y Ardón (2007) en base a la disponibilidad de estos residuos en cada comunidad (Tabla 1).

Los sustratos fueron picados a un tamaño de partícula de 2 a $3 \mathrm{~cm}$, posteriormente se mezclaron de acuerdo a las formulaciones y se colocaron en sacos malla. Al respecto, Sánchez \& Royse (2002), manifiesta que los materiales elegibles para ser utilizados en la preparación de sustratos deben poseer el mayor y mejor número posible de propiedades positivas tales como la buena disponibilidad en cantidad y continuidad, conocimiento de sus características físico-químicas, localización cercana y facilidad de transporte y manejo, buena interrelación cepa-sustrato que debe respetarse para obtener rendimientos óptimos.

Pasteurización

Para el cultivo de Pleurotus, la pasteurización de los sustratos se llevó a cabo utilizando sacos malla que fueron sometidos a inmersión en agua a $80^{\circ} \mathrm{C}+/-1$ por 1 hora; este proceso elimina parcialmente los microorganismos ambientales presentes en el sustrato (Rojas, 2004), con temperaturas superiores se corre el riesgo de modificar la composición química del material limitando un aprovechamiento eficaz de las fuentes de carbono por el micelio del hongo; además, los azúcares disueltos se hacen accesibles a otros microorganismos contaminantes, que los pueden consumir con mayor facilidad y rapidez.

En el caso de L. edodes el sustrato formulado se colocó en bolsas de polietileno de 10 x 15 x 2 cm con 2 $\mathrm{kg}$ de capacidad, las que fueron pasteurizadas con vapor de agua utilizando cilindros de $200 \mathrm{~kg}$ durante 5 horas a $65{ }^{\circ} \mathrm{C}$.

Siembra e incubación

Una vez pasteurizado, atemperado $\left(20{ }^{\circ} \mathrm{C}\right)$ y drenado, el sustrato para Pleurotus quedó apto para la siembra con inóculo fúngico a razón de $50 \mathrm{~g}$ por unidad de cultivo, colocándolos en bolsas de polietileno de 14 x 23 x $2 \mathrm{~cm}$ de $5 \mathrm{~kg}$ de capacidad. Luego de la inoculación se hicieron cortes de $2 \mathrm{~cm}$ de largo en toda la superficie. En L. edodes, la siembra se realizó en presencia de un mechero de alcohol utilizando $10 \mathrm{~g}$ de inóculo fúngico por cada unidad de cultivo. Todas las unidades fueron incubadas a temperaturas entre 20 a 25 ${ }^{\circ} \mathrm{C}$ hasta completar el desarrollo miceliar y sin iluminación (Romero et al., 2000; García, 2003; Fernández, 2004) (Figura1).

Fructificación - Inducción de la formación de primordios

Finalizada la incubación se realizó el cambio de las condiciones ambientales para iniciar la etapa de inducción a la formación de primordios. Las unidades de cultivo de Pleurotus se trasladaron al área de fructificación a temperatura ambiente; en esta etapa se incrementó la humedad relativa con riego constante. En el caso de $L$. edodes luego del amarronamiento, la inducción se realizó mediante inmersión en agua fría durante 24 h y se trasladó al ambiente de fructificación para el desarrollo de los basidiocarpos.

Evaluación de la productividad

Los basidiocarpos obtenidos en cada comunidad fueron cosechados en un estado de madurez óptimo, considerando el nivel de curvatura del borde del píleo (Sánchez \& Royse, 2001). Los indicadores de la productividad evaluados fueron: la eficiencia biológica (EB), el ciclo de cultivo (CC) y la tasa de productividad (TP) mediante las fórmulas especificadas en la Tabla 2.

Tabla 2. Indicadores de Producción.

\begin{tabular}{ll}
\hline INDICADOR & FÓRMULA \\
\hline EB & Kg hongo (pesos fresco) \\
\cline { 2 - 2 } (Eficiencia Biológica) & Kg de sustrato (peso seco) \\
CC & Días desde la inoculación \\
(Ciclo de Cultivo) & hasta la última cosecha \\
TP & $\frac{E B}{C C}$ \\
(Tasa de Productividad) & \\
\hline
\end{tabular}

Fuente: Reyes et al., 2004.

\section{Evaluación de Parámetros Ambientales}

Se consideraron los factores físicos de temperatura y humedad relativa como indicadores determinantes para la fase de fructificación; los valores de estos factores fueron registrados durante los meses de enero a junio del 2016 (ciclo de cultivo de las tres especies), tales como los máximo y mínimo, semanalmente. Para este propósito se usaron termohigrometros digitales Boeco Htc-1 colocados en cada ambiente de cultivo para las tres comunidades campesinas. Los valores obtenidos fueron promediados y se calculó la variación estándar (VS) para cada comunidad.

Análisis Fisicoquímico

Los basidiocarpos se colectaron en los centros de producción de forma aleatoria para ser trasladados a los laboratorios de la Unidad de Prestaciones de Servicio de Análisis Químico (Facultad de Ciencias UNSAAC) en donde se realizaron los análisis de aminoácidos mediante el sistema Pico Tag (Water. USA). 


\section{Resultados y discusión}

Los rangos de temperatura promedio de las tres comunidades fluctuaron entre 12 y $14.8{ }^{\circ} \mathrm{C}$ (Tabla 3) favoreciendo la formación de P. ostreatus y L.edodes con rangos de temperatura más amplios que los reportados por Albertó (2008), quien menciona rangos de entre $15-18{ }^{\circ} \mathrm{C}$ para ambas especies, resultados que nos muestran la adaptabilidad de estos hongos a bajas temperaturas; mientras que la humedad relativa se mantuvo casi constante en las tres comunidades (85\%), valores óptimos para esta etapa del cultivo e indispensable para la estimulación de la formación de primordios (Pérez, 1996; Albertó, 2008).

Tabla 3. Temperatura y humedad relativa promedio del área de fructificación para Pleurotus y Lentinula en las tres comunidades.

\begin{tabular}{lll}
\hline \multirow{2}{*}{ COMUNIDAD } & \multicolumn{2}{c}{ FRUCTIFICACIÓN } \\
\cline { 2 - 3 } & $\mathrm{T}^{\circ} \mathrm{y}$ VS & $\mathrm{HR}$ \\
\hline HARIN & $14.8+/-1$ & $85+/-1$ \\
HUAYLLAY & $13.5+/-4$ & $85+/-1$ \\
SAN NICOLAS DE BARI & $12.0+/-1$ & $84+/-1$ \\
\hline
\end{tabular}

$\mathrm{T}^{\circ}$ : Temperatura en ${ }^{\circ} \mathrm{C}$

VS: Variación estándar.

HR: Humedad relativa en $\%$.

Tabla 4. Indicadores de producción de las tres especies en estudio.

\begin{tabular}{|c|c|c|c|c|}
\hline ESPECIE & COMUNIDAD & $\mathrm{CC}$ & $\begin{array}{l}\text { EB } \\
\%\end{array}$ & $\begin{array}{l}\mathrm{TP} \\
\%\end{array}$ \\
\hline P. ostreatus & Huayllay & 91.0 & 55.8 & 0.6 \\
\hline P. ostreatus & San Nicolás de Bari & 75.0 & 43.8 & 0.6 \\
\hline P. ostreatus & Harin & 42.0 & 58.2 & 1.4 \\
\hline P. djamor & Huayllay & --- & --- & --- \\
\hline P. djamor & San Nicolás de Bari & 90.0 & 34.8 & 0.4 \\
\hline P. djamor & Harin & 42.0 & 27.3 & 0.7 \\
\hline L.. edodes & Huayllay & --- & --- & --- \\
\hline L. edodes & San Nicolás de Bari & 135.0 & 51.3 & 0.4 \\
\hline L. edodes & Harin & 155.0 & 40.6 & 0.3 \\
\hline
\end{tabular}

P. ostreatus presentó EB entre $43.8 \%$ y $58.2 \%$, con ciclos de cultivo relativamente cortos de entre 42 y 75 días en las comunidades de Harin y San Nicolás de Bari, respectivamente; mientras que, en Huayllay los ciclos fueron más largos, hasta de 91 días (Tabla 4), debido probablemente a las mayores fluctuaciones de temperatura que se presentaron en esta comunidad (VS = 4, Tabla 3). Al respecto, Albertó (2008) menciona que la disminución de la temperatura desacelera el desarrollo de las fructificaciones pero, los hongos se forman con estructura más firme. Por su parte, Vogel \& Salmones (2000) refieren que algunas cepas de $P$. ostreatus presentan una temperatura optima de alrededor de $19{ }^{\circ} \mathrm{C}$, encontrando estos autores que, cuando se mantiene la temperatura adecuada para cada especie, estas generan mayores valores de EB. En cuanto a $P$. djamor y $L$. edodes, estas cepas se adaptaron satisfactoriamente en las comunidades de Harin y San Nicolás de Bari, con EB que fluctuaron entre $34 \%$ y $51 \%$, respectivamente, para cada especie. Mientras, las bajas temperaturas fueron un factor limitante para la fructificación de estas especies en la comunidad de Huayllay, donde el desarrollo miceliar

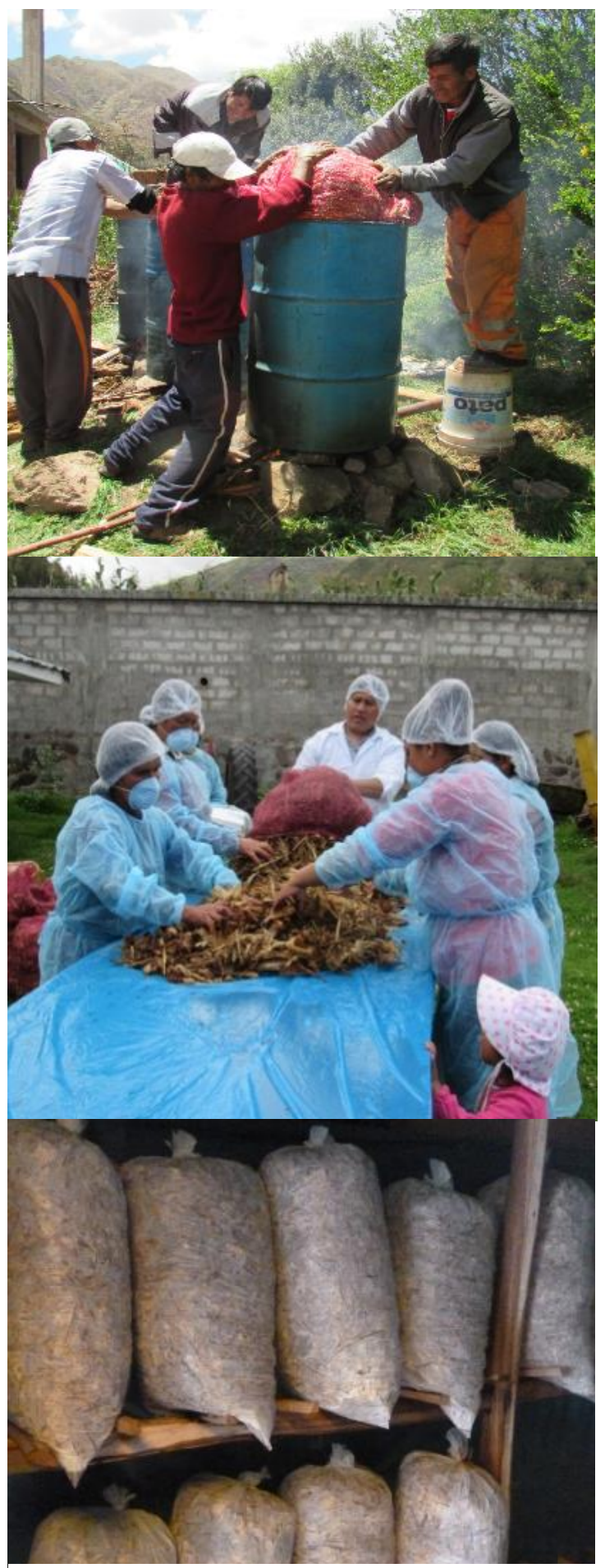

Figura 1. Cultivo de Pleurotus spp. y Lentinula edodes en las comunidades campesinas (Pasteurización, inoculación e Incubación). 
Tabla 5. Análisis fisicoquímico de los hongos cultivados en las tres comunidades.

\begin{tabular}{|c|c|c|c|c|c|c|c|}
\hline COMUNIDAD & Huayllay & San Nico & s de Bari & & Harin & & \\
\hline ESPECIE & 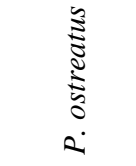 & 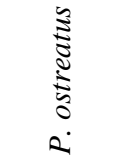 & 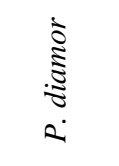 & 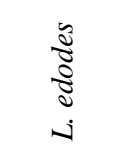 & 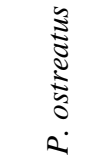 & 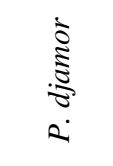 & 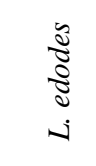 \\
\hline HUMEDAD (\%) & 90.72 & 91.65 & 84.83 & 14.1 & 89.2 & 13.3 & 86.31 \\
\hline Proteínas (\%) & 27.11 & 35.45 & 14.02 & $24 ., 21$ & 17.76 & 19.68 & 34.54 \\
\hline Grasa $(\%)$ & 2.05 & 4.31 & 2.19 & 2.79 & 3.12 & 2.66 & 2.31 \\
\hline Ceniza (\%) & 7.02 & 10.66 & 5.41 & 7.21 & 8.06 & 7.73 & 10.28 \\
\hline Fibra $(\%)$ & 13.50 & 14.85 & 9.13 & 11.87 & 13.5 & 12.00 & 8.17 \\
\hline Carbohidratos (\%) & 63.82 & 49.58 & 78.38 & 65.79 & 71.06 & 69.93 & 52.87 \\
\hline Calcio (mg/100 g) & 3.5 & 4.6 & 4.4 & 24 & 37.5 & 36.1 & 5.1 \\
\hline Magnesio (mg/100 g) & 12 & 16.2 & 15.2 & 92.7 & 122.2 & 97.8 & 18.1 \\
\hline Fosforo $(\mathrm{mg} / 100 \mathrm{~g})$ & 124 & 130 & 104 & 655 & 719 & 682 & 110 \\
\hline Hierro (mg/100 g) & 1.1 & 1.6 & 0.9 & 7 & 8.9 & 5.4 & 0.8 \\
\hline Zinc (mg/100 g) & 0.33 & 0.28 & 0.26 & 1.6 & 2.24 & 2.1 & 0.17 \\
\hline
\end{tabular}

sobre el sustrato se vio afectado, evitando obtener resultados positivos para estos tratamientos. Según Ríos et al. (2010), las eficiencias biológicas a partir del $50 \%$ se consideran aceptables para una producción económicamente rentable, por lo que, los valores reportados nos permiten inferir la factibilidad de su cultivo a pequeña escala en las comunidades del ámbito de estudio. Además, $P$. ostreatus, $P$. djamor y L. edodes son hongos versátiles que se adaptan a las condiciones ambientales de las comunidades del ámbito de estudio, los mismos que se pueden cultivar bajo condiciones artesanales, llegando a formar parte de sus actividades agropecuarias (Figuras 1 y 2). Al respecto Zharare et al. (2010) mencionan que el cultivo de hongos se ha convertido en una estrategia mundial para disminuir la pobreza y diversificar la producción agrícola; por otro, lado Cardona (2011) afirma que el cultivo casero de hongos en países de Centroamérica ha tenido gran aceptación entre las familias, ya que este contribuye a la producción y disponibilidad de alimentos, no requiere altas inversiones de dinero, pudiendo ser una fuente de ingresos extra para las familias.

El contenido de proteínas en peso seco de $P$. ostreatus y L. edodes alcanzaron los valores más altos, $35.45 \%$ y $34.54 \%$, en las comunidades de San Nicolás de Bari y Harin, respectivamente (Tabla 5). Al respecto, Diez \& Alvarez (2001) y Manzi et al. (2004) indican que el contenido proteico de los hongos oscila entre $15 \%$ y $35 \%$ de peso seco, dependiendo de las especies, de las variedades y de la etapa de desarrollo del cuerpo fructífero. Así, aunque el contenido de proteínas de los hongos cultivados sean menores al de las carnes animales, los valores superan al de los vegetales convencionales v.g. arroz (7.3\%), trigo (13.2\%) (Mshigeni \& Chang, 2017). Además, son una buena fuente de carbohidratos no amiláceos, alta cantidad de fibra, aminoácidos, minerales y vitaminas (Ahmed et al., 2013). En el Perú son apreciados en gastronomía desde tiempos pre-hispánicos (Trutmann, 2012).

Tabla 6. Comparación del contenido de minerales y proteínas de Pleurotus ostreatus, $P$. djamor y Lentinula edodes en relación a otros alimentos (mg/100g).

\begin{tabular}{|c|c|c|c|c|c|c|c|c|c|}
\hline MINERAL & 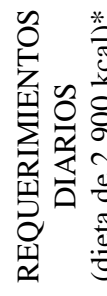 & 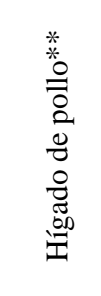 & 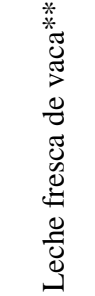 & 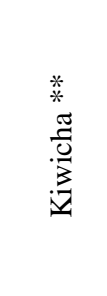 & $\begin{array}{l}* \\
\stackrel{*}{*} \\
\stackrel{5}{\sigma} \\
\stackrel{⿹}{*}\end{array}$ & 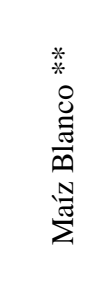 & 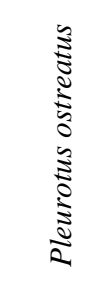 & $\begin{array}{l}\grave{\vdots} \\
\vdots \\
\vdots \\
\dot{0} \\
2\end{array}$ & $\begin{array}{l}\mathfrak{z} \\
\mathbb{Z} \\
\mathbb{z} \\
j \\
j\end{array}$ \\
\hline $\mathrm{P}$ & 800 & 272 & 24 & 453 & 123 & 249 & 719 & 682 & 655 \\
\hline $\mathrm{Ca}$ & 800 & 11 & 106 & 236 & 30 & 5 & 37.5 & 36.1 & 24 \\
\hline $\mathrm{Fe}$ & 10 & 8.56 & 1.3 & 7.32 & 1.4 & 1.48 & 8.9 & 5.4 & 7 \\
\hline $\mathrm{Zn}$ & 15 & 3.07 & 0.4 & 2.68 & 1.38 & 1.91 & 2.24 & 2.1 & 1.6 \\
\hline Proteína & - & 18 & 3.1 & 12.8 & 11.6 & 5.9 & 35.45 & 19.68 & 34.54 \\
\hline
\end{tabular}




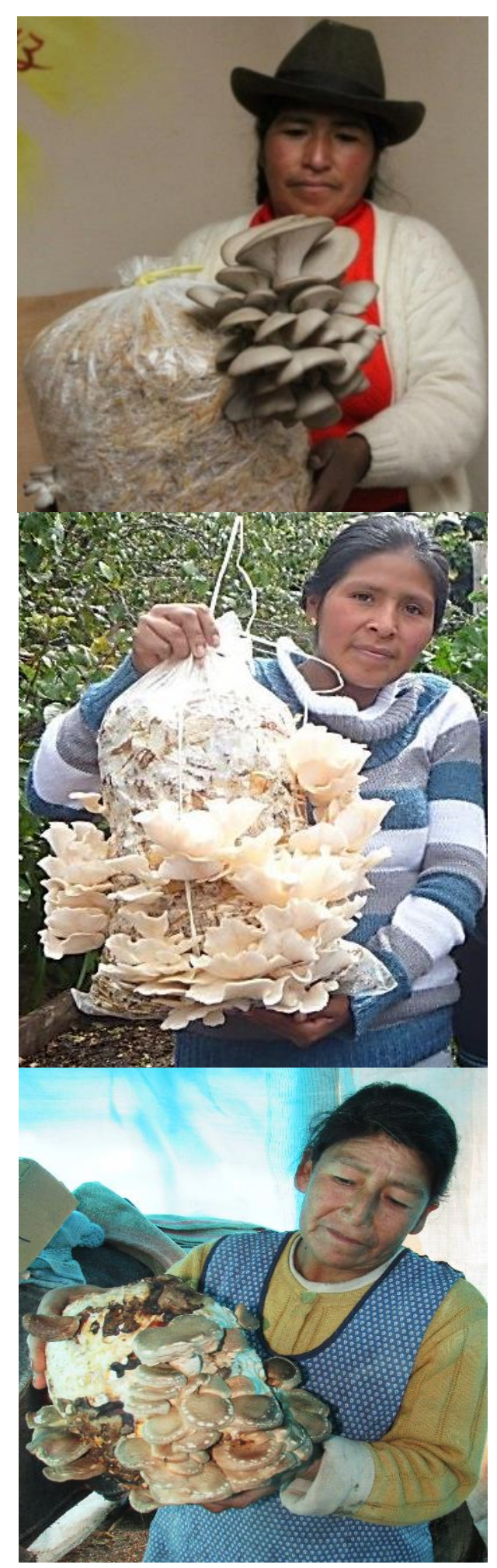

Figura 2. Basidiocarpos de $P$. ostreatus, $P$. djamor y L. edodes obtenidas en las comunidades campesinas de Huayllay, San Nicolás de Bari y Harin.
Al comparar los resultados con los alimentos de consumo diario se observa que los hongos en estudio son una importante fuente de minerales y proteínas, principalmente fosforo, magnesio, calcio y hierro (Tabla 6). Según Manzi et al. (1999), los hongos cultivados contienen una cantidad razonable de macroelementos y microelementos.

Tabla 7. Composición de aminoácidos de Pleurotus ostreatus.

\begin{tabular}{cccc}
\hline & & & \\
COMUNIDAD & $\mathrm{mg} / \mathrm{g}$ & $\mathrm{mg} / \mathrm{g}$ & $\mathrm{mg} / \mathrm{g}$ \\
\cline { 2 - 4 } & 28.20 & 30.20 & 31.9 \\
AMINOACIDOS & 34.40 & 36.40 & 35.4 \\
Asp & 24.80 & 24.80 & 26 \\
Glu & 48.30 & 50.00 & 52.9 \\
Ser & 8.60 & 9.20 & 10.3 \\
Gly & 42.20 & 43.20 & 43.8 \\
His & $34 ., 00$ & 29.00 & 31.2 \\
Arg & 36.30 & 32.60 & 36.7 \\
Thr & 22.90 & 22.20 & 25.3 \\
Ala & 42.80 & 45.90 & 43.6 \\
Pro & 13.50 & 15.80 & 14.3 \\
Tyr & 9.90 & 11.80 & 11.8 \\
Val & 35.70 & 40.50 & 27.5 \\
Met & 17.70 & 20.40 & 19.5 \\
Cys & 22.10 & 24.30 & 24 \\
Ile & 27.30 & 27.30 & 25.5 \\
Leu & 51.10 & 36.30 & 40.3 \\
Phe & & & \\
Lys & & & \\
& & &
\end{tabular}

$P$. ostreatus cultivado en las tres comunidades presenta valores apreciables de aminoácidos, encontrándose en ellos ocho de los diez aminoácidos esenciales requeridos para una alimentación saludable. Manzi et al. (2004) mencionan que los hongos son relativamente altos en proteínas de buena calidad y contienen todos los aminoácidos esenciales para la nutrición humana, principalmente leucina y lisina, carente en la mayoría de los cereales. Según la Organización Mundial de la Salud (OMS), los champiñones son especialmente ricos en ácido glutámico, ácido aspártico y arginina; de igual forma, Manzi et al. (1999) señalan que los aminoácidos más abundantes en $P$. ostreatus, $P$. eryngii, $P$. pulmunarius y $L$. edodes cultivados en paja de trigo con un $15 \%$ de remolacha azucarera, expresados como porcentajes de aminoácidos totales, son: el ácido glutámico (12.8$20.9 \%$ ), el ácido aspártico (9.1-12.1\%) y la arginina (3.7-11.7\%). Comparando estos valores con los resultados obtenidos (Tabla 7), P. ostreatus cultivado en las comunidades campesinas de Harin, San Nicolás de Bari y Huayllay parece ser el que mayor calidad proteica presenta; algunas de las variedades de esta especie tienen una buena composición, tanto de aminoácidos esenciales como de no esenciales (Dundar 
et al., 2008; Patil et al., 2010). La composición en aminoácidos de las proteínas de los hongos es comparable a la de la proteína animal, lo cual sería importante hoy en día para hacer frente al alto consumo de alimentos proteicos de origen animal, sobre todo en los países desarrollados (Guillamón et al., 2010).

\section{Conclusiones}

De las tres especies cultivadas, Pleurotus ostreatus presenta índices de producción aceptables para un cultivo a baja escala (EB entre $43.8 \%$ y $58.2 \%$, y TP entre 0.6 y 1.4), siendo capaz de adaptarse a las diferentes condiciones ambientales de las comunidades campesinas estudiadas.

El análisis proximal de proteínas y aminoácidos de los basidiocarpos se encuentra dentro de los parámetros reportados por otros autores, destacando P. ostretus, que presenta valores considerables de proteína, fibra, macro, micronutrientes y la presencia de 17 aminoácidos, de los cuales ocho son esenciales.

\section{Agradecimientos}

Al Dr. Daniel Martínez-Carrera por el constante apoyo brindado en la ejecución del presente estudio e invitarnos a ser parte de la Red Latinoamericana de Hongos Comestibles y Medicinales; de igual forma al Vicerrectorado de Investigación de la UNSAAC por el financiamiento del estudio a través de los Fondos Canon. Al Dr. Luis A. Ponce Soto, por los análisis de aminoácidos, y a la Unidad de Prestaciones de Servicio de Análisis Químico (Facultad de Ciencias UNSAAC) por los análisis fisicoquímicos.

\section{Literatura citada}

Ahmed M., Abdullah N., Ahmed K. \& Bhuyan M. 2013. Yield and nutritional composition of oyster mushroom strains newly introduced in Bangladesh. Pesquisa Agropecuaria Brasileira, 48(2): 197-202. DOI: http://dx.doi.org/10.1590/S0100-204X2013000200010.

Albertó E. 2008. Cultivo intensivo de los hongos comestibles: como cultivar champiñones, gírgolas, shiitake y otras especies. Hemisferio Sur. Buenos Aires.

Ardón C. 2007. La producción de los hongos comestibles. Tesis Mg Ed. Guatemala. USCG.

Cardona A. 2011. Colección Buenas Practicas, Hongos tipo ostra. FAO (Organización de las Naciones Unidas para la Alimentación y la Agricultura).

Chang S.T. 1993. Mushroom Biology: The impact on mushroom production and mushroom products. Chang, Buswell and Chiu Eds. Mushroom Biology and Mushroom Products. Proceedings of the First International Conference on Mushroom Biology and Mushroom Products. The Chinese University Press. Hong Kong.
Chimey C. \& Holgado M. 2010. Los hongos comestibles Silvestres y Cultivados en Perú. En: Martínez-Carrera D., Curvetto N., Sobal M., Morales P. \& Mora V.M. Hacia un Desarrollo Sostenible del Sistema de ProducciónConsumo de los Hongos Comestibles y Medicinales en Latinoamérica: Avances y perspectivas en el siglo XXI. 381-395. Red Latinoamericana de Hongos Comestibles y Medicinales.

Da Silva M., Naozuka J., da Luz J., de Assunção L., Oliveira P., Vanetti M., Bazolli D. \& Casuya M. 2012. Enrichment of Pleurotus ostreatus mushrooms with selenium in coffee husks. Food Chemistry, 131(2): 558-563. DOI: https://doi.org/10.1016/j.foodchem.2011.09.023.

Diez V.A. \& Alvarez A. 2001. Compositional and nutritional studies on two wild edible mushrooms from northwest Spain. Food Chemistry, 75: 417-422.

Dundar A., Acay H. \& Yildiz A. 2008. Yield performances and nutritional contents of three oyster mushroom species cultivated on wheat stalk. African Journal of Biotechnology, 7: 3497-3501.

Fernández F. 2004. Guía práctica de producción de Setas (Pleurotus spp.). Fungitec Asesorías.Guadalajara, Jalisco, Mexico.

García R.M. 2003. Cultivo de setas y trufas. Ediciones Mundi-Prensa. Madrid - España.

Guillamón E., García-Lafuente A., Lozano M., D’Arrigo M., Rostagno M., Villares A. \& Martínez J.A. 2010. Edible mushrooms: role in the prevention of cardiovascular diseases. Fitoterapia, 81: 715-723. DOI: https://doi.org/10.1016/j.fitote.2010.06.005.

Herrera F.L. 1930. Plantarum cuzcorum herrerarianum: Estudios sobre la Flora del Departamento del Cuzco. Vol. 1. San Marti y Cia. Lima.

Manzi P., Gambelli L., Marconi S., Vivanti V. \& Pizzoferrato L. 1999. Nutrients in edible mushrooms: an inter-species comparative study. Food Chemistry, 65: 477-482.

Manzi P., Marconi S., Aguzzi A. \& Pizzoferrato L. 2004. Commercial Mushrooms: Nutritional quality and effect of cooking. Food Chemistry, 84: 201-206.

Martínez-Carrera D., Curveto M., Sobal M., Morales P. \& Mora V.M. 2010. Hacia un Desarrollo Sostenible del Sistema de Producción-Consumo de los Hongos Comestibles y Medicinales en Latinoamérica. Avances y Perspectivas en el siglo XXI. Red Latinoamericana de Hongos Comestibles y Medicinales.

Martínez-Carrera D., Quirarte M., Soto Velazco C., Salmones D. \& Guzmán G. 1984. Perspectiva sobre el cultivo de hongos comestibles en residuos agroindustriales en México. Bol. Soc. Mex. Mic., 19: 207-219.

MINAM (Ministerio del Ambiente, Perú). 2015. Servicio de Prospección, Colección, Elaboración de Mapas de Distribución y Estudio Socioeconómico De Conocimientos Tradicionales Asociados al Cultivo de las Razas de Maíz. Equipo Consultor ASPROMAD. Lambayeque-Perú.

Mshigeni K. \& Chang S. 2017. Hongos, Medio Ambiente y Salud Humana. Tesoros de la tierra para la salud, la felicidad y la longevidad. Guatemala, América Central: Centro Editorial Vile.

Patil S.S., Ahmed S.A., Telang S.M. \& Baig M.M. 2010. The nutritional value of Pleurotus ostreatus (Jacq. Fr) Kumm cultivated on different lignocellulosic agrowastes. Innovative Romanian Food Biotechnology, 7: 66-76. 
Pérez G.E. 1996. Producción de hongos comestibles (Setas y Champiñones). Centro de Investigaciones Sociales, Tecnológicas y Agroindustriales de la Agricultura Mundial (CIESTAAM) 2da. Edición. México.

Pérez-Merlo R. \& Mata G. 2005. Cultivo y selección de cepas de Pleurotus ostreatus y $P$. pulmonarius en viruta de pino: obtención de nuevas cepas y evaluación de su producción. Revista Mexicana de Micología, 20: 53-59.

Reyes G.R., Abella A.E., Eguchi F., LijimaT., Higaki M., Quimio T.H. 2004. Growing paddy Straw mushroom. In: Mushroom grower's handbook 1; Oyster mushroom cultivation. 262-269. Mushroom World. Corea.

Reyes M., Gómez-Sánchez I., Espinoza C. \& Bravo F. 2009. Tablas peruanas de composición de alimentos. Ministerio de Salud, Instituto Nacional de Salud. Lima, Perú.

Ríos M. del P., Hoyos J.L. \& Mosquera S.A. 2010. Evaluación de los parámetros productivos de la semilla de Pleurotus ostreatus propagada en diferentes medios de cultivo. Biotecnología en el Sector Agropecuario y Agroindustrial, 8: 86-94.

Rojas E.A. 2004. Evaluación de paja de trigo (Triticum sativum); broza de encino, (Quercus sp.) y rastrojo de maíz, (Zea mays) para el cultivo del hongo comestible Pleurotus ostreatus bajo condiciones artesanales en San Rafael la Independencia, Huehuetenango. Tesis de grado. Universidad San Carlos de Guatemala.

Romero J., Rodríguez M. \& Perez R. 2000. Pleurotus ostreatus: Importancia y tecnología del cultivo. Grupo de Nutrición, Departamento de Física-Química / Facultad de Mecánica / Universidad de Cienfuegos Carlos Rafael Rodríguez. Cuatro caminos. Ciudad de Cienfuegos. Cuba.
Roncero Ramos I. 2015. Propiedades Nutricionales y saludables de los hongos. 12-23. Centro Tecnológico de Investigación del Champiñón de la Rioja (CETICH). La Rioja, España.

Sánchez C. 2004. Modern aspects of mushroom culture technology. Applied Microbiol Biotechnol., 64(6): 756762. DOI: https://doi.org/10.1007/s00253-004-1569-7.

Sánchez J. \& Royse D. 2001. La biología y el cultivo de Pleurotus spp. UTEHA Noriega Editores. México.

Sánchez Vásquez J.E. \& Royse D. 2002. La Biología y el Cultivo de Pleurotus spp. LIMUSA, S. A. México.

Trutmann P. 2012. The Forgotten Mushrooms of Ancient Peru. Global Mountain Action, 33.

Vega A., Caballero R.E., García J.R. \& Mori N. 2005. Bioconversion of agroindustrial residues by Pleurotus ostreatus cultivation. Revista Mexicana de Micología, 20: 33-38. Sociedad Mexicana de Micología, Xalapa, México.

Vogel F. \& Salmones D. 2000. Análisis comparativo de la productividad de cepas de Pleurotus spp. cultivadas en una planta comercial. Revista Iberoamericana de Micología, 17: 138-141. Veracruz, MX.

Zharare G., Kabanda S. \& Poku J. 2010. Effects of temperature and hydrogen peroxide on mycelial growth of eight Pleurotus strains. Scientia Horticulturae, 125: 95102.

\footnotetext{
${ }^{1}$ Escuela profesional de Biología / Facultad de Ciencias / Universidad Nacional de San Antonio Abad del Cusco (UNSAAC). Cusco / Perú. Autor de correspondencia: Tel.: +84226039, Cel: 974244940, e-mails: maria.holgador@unsaac.edu.pe; mariholgado@yahoo.es.

${ }^{2}$ Centro de Investigación y producción de Hongos Alimenticios y Medicinales - CIPHAM.

${ }^{3}$ Escuela profesional de Química / Facultad de Ciencias / Universidad Nacional de San Antonio Abad del Cusco (UNSAAC). Cusco / Perú.
} 